\title{
1 Listening to bacterial Esperanto: transcriptome reprogramming in a
}

\section{2 plant beneficial rhizobacterium}

3 Ana Bejarano ${ }^{1,2 *}$, Michele Perazzolli ${ }^{1,2}$, Ilaria Pertot ${ }^{1,2}$, Gerardo Puopolo $^{1,2}$

4

$5 \quad{ }^{1}$ Center of Agriculture, Food, Environment, University of Trento, San Michele all'Adige, Italy

$6{ }^{2}$ Department of Sustainable Agro-Ecosystems and Bioresources, Research and Innovation Centre,

7 Fondazione Edmund Mach, San Michele all'Adige, Italy

8 *corresponding author: Ana Bejarano (ana.bejaranoramos@unitn.it) 


\section{Abstract}

Intraspecies, interspecies and interkingdom signalling occurring via diffusible communication signals (DCSs) continuously shapes the gene expression patterns of individual bacterial species in the rhizosphere, affecting bacterial functions within the rhizosphere microbial community. To unravel how DCSs influence rhizosphere competence of plant beneficial rhizobacteria, we carried out a functional and transcriptome analysis on the plant beneficial bacterium Lysobacter capsici AZ78 (AZ78). Results reveal that 13-methyltetradecanoic acid and indole, glyoxylic acid and 2,3butanedione play a relevant role in the interaction between $L$. capsici members and other soil-living (micro)organisms. DCSs regulated mechanisms of multistress and multidrug tolerance and persistence, including detoxification, motility, antibiotic production, and expression of secretion systems. In particular, 13-methyltetradecanoic acid, glyoxylic acid and 2,3-butanedione might enable AZ78 to rapidly colonize the rhizosphere. Moreover, glyoxylic acid and 2,3-butanedione elicit biological responses to outcompete other (micro)organisms. In contrast, indole inactivates twitching motility and antibiotic production. These results demonstrate that DCSs influence the functioning of plant beneficial rhizobacteria and suggest that plant beneficial $L$. capsici strains could use them to foster rhizosphere colonization and enhance in vivo activities to increase soil health and plant fitness.

Keywords: diffusible communication signals, rhizosphere, plant beneficial bacteria, bacterial interactions, transcriptome, indole, diffusible signal factors, glyoxylic acid 


\section{Introduction}

Rhizosphere microbial communities play a key role in soil fertility and in controlling soil-borne phytopathogenic microorganisms [1]. Their composition and functioning relay on root exudates and signalling molecules governing the complex interactions occurring between microorganisms and plants [2]. Bacterial intraspecies and interspecies communication occur mainly via Diffusible Communication Signals (DCSs) that allow bacterial communities to form and synchronize their behaviour [2]. N-acyl homoserine lactones (AHLs), produced by Gram-negative plant-associated bacteria, are among the most studied DCSs [3, 4]. Several works showed that endogenous and exogenous AHLs play an essential role in multiple bacterial physiological and biochemical behaviour [5]. Diffusible Signal Factors (DSFs) is another subgroup of DCSs produced by Gram-negative bacteria and it was initially reported in the phytopathogenic bacterium Xanthomonas campestris pv. campestris (Xcc) [6]. DSFs have been identified in other bacterial species as well and they have been linked to virulence, motility, biofilm production, and extracellular enzyme production [7]. Besides DSFs, Xanthomonadaceae family use diffusible factors (DFs) as DCSs; these signals are involved in the regulation of secondary metabolites biosynthesis and antioxidant activity [8-11]. In addition, plant-associated bacteria produce volatile organic compounds (VOCs) that are involved in communication and competition between physically separated soil microorganisms [12]. Among VOCs, indole (IND) is an ubiquitous interkingdom signal that influences antibiotic resistance, motility, biofilm formation, and virulence, and has the potential to be a DCS [13]. Other VOCs mediating changes in gene expression related to motility and antibiotic resistance are 2,3-butanedione (BUT) and glyoxylic acid (GLY) [14].

Among plant-associated Gram-negative bacteria, Lysobacter spp. belonging to the Xanthomonadaceae family, are commonly found in the plant rhizosphere where they control phytopathogenic microorganisms [15]. This ability mainly relies on the release of lytic enzymes and antibiotics like the Heat Stable Antifungal Factor (HSAF), which are toxic to phytopathogenic 
(micro)organisms [16, 17]. In L. enzymogenes DSFs, DFs and IND regulate HSAF biosynthesis [9, 18, 19] and twitching motility [20]. Furthermore, IND reverses the intrinsic antibiotic resistance of L. enzymogenes through the two-component regulatory system QseC/QseB [19, 20]. However, with the only exception of the involvement of DSFs and AHLs in L. brunescens behaviour [21], a complete overview of the overall effect of DCSs in Lysobacter spp. has not been described yet. In particular, it is not known if intra-, interspecies and interkingdom DCSs have a role in the establishment of Lysobacter spp. in the plant rhizosphere and if DCSs influence Lysobacter spp. to form stable communities with resident (micro)organisms or its ability to control phytopathogenic microorganisms.

To clarify these questions, we carried out a functional and transcriptome analysis of the plant beneficial bacterium L. capsici AZ78 (AZ78) in presence of a wide range of DCSs (13methytetradecanoic acid [LeDSF3], IND, GLY, BUT, 3-hydroxybenzoic acid [3HBA], 4hydroxybenzoic acid [4BHA], N-[3-hexanoyl]-L-homoserine lactone, N-[3-oxooctanoyl]-Lhomoserine lactone, and $\mathrm{N}$-[3-oxododecanoyl]-L-homoserine lactone). Genes encoding proteins involved in cell-cell communication systems were identified through genome mining. Functional experiments aimed to the assess changes in the biological properties of AZ78 upon exposure to DCSs and paid particular attention to bacterial growth and the ability of AZ78 to inhibit plant pathogens. Simultaneously, gene expression profiling of AZ78 exposed to main DCSs was carried out by high throughput RNA-Seq.

\section{Materials and methods}

\section{Microorganisms and diffusible communication signals}

Bacterial strains (Table S1) were routinely grown on Nutrient Agar (Oxoid, Basingstoke, UK) at $27^{\circ} \mathrm{C}$. The phytopathogenic oomycete Pythium ultimum was maintained on Potato Dextrose Agar (Oxoid) at $25^{\circ} \mathrm{C}$. 
LeDSF3 was obtained from Avanti Polar Lipids (Alabaster, Alabama, USA). IND, GLY, BUT, 3HBA, 4BHA, N-(3-hexanoyl)-L-homoserine lactone, N-(3-oxooctanoyl)-L-homoserine lactone; and N-(3-oxododecanoyl)-L-homoserine lactone were purchased from Merck (Sigma-Aldrich, Darmstadt, Germany). Aqueous stock solutions were prepared, except for LeDSF3 and the mixture of AHLs that were prepared in pure methanol.

\section{Genome mining}

AZ78 genome was mined to identify putative genes involved in cell-cell communication systems using nucleotide and protein sequence comparison. Genes from L. enzymogenes C3, Stenotrophomonas maltophilia (Sm) K279a and Xcc ATCC $33913^{\mathrm{T}}$ were aligned against AZ78 genome, using RAST [22] to identify putative AZ78 genes responsible for DCS synthesis, reception and regulation using a cut-off of $1 \times 10^{-5}$ at amino acid level. Putative genes were analysed with BLASTP [23], and length $>70$ and identity $>70 \%$ at amino acid level were used as threshold. Identified gene clusters encoding putative proteins involved in cell-cell communication systems in AZ78 were then used to mine the Lysobacter spp. genomes, following the methodology described above. All genomes were downloaded from the National Center for Biotechnology Information (NCBI) (https://www.ncbi.nlm.nih.gov/) (Table S1). For the phylogenetic analyses, nucleotide sequences were aligned using ClustalW [24]. Evolutionary distances were assessed by applying Kimura's two-parameter model [25] and the best phylogenetic trees were inferred by neighbourjoining method [26] implemented in MEGA 7 [27]. Confidence values for nodes in the trees were generated by bootstrap analysis [28] using 1000 permutations of the data sets.

\section{Assessment of diffusible communication signal production}

Production of AHLs by AZ78 and Lysobacter spp. type strains was assessed by evaluating their ability to restore violacein production in Chromobacterium violaceum CV026 and/or to promote lacZ transcription in Agrobacterium tumefaciens NT1 (pZLR4) as previously described [29, 30]. Likewise, 
the ability to release DSF was determined using the bacterial reporter strain Xcc 8523 pL6engGUS according to Slater et al., 2000 [31]. Pseudomonas chlororaphis M71 [32] was used as an AHL positive control, whereas Xcc 8004 was used as a DSF positive control [6]. For each condition, five replicates were used, and the experiment was repeated.

\section{Evaluation of diffusible communication signal effect on cell growth}

The effect of DCSs on AZ78 cell growth rate was assessed on 1/10 Tryptic Soy Broth (Oxoid) amended with each DCS (Table S2). AZ78 (starting concentration $1 \times 10^{7} \mathrm{CFU} / \mathrm{mL}$ ) was grown at $27^{\circ} \mathrm{C}$ on a 96-well plate $(200 \mu \mathrm{L})$ and absorbance at $600 \mathrm{~mm}$ was recorded on a microplate reader (Synergy 2 Multi-Mode Microplate Reader, BioTek, Winooski, Vermont, USA). Non-inoculated media were used as blank. For each condition, five replicates were used. The experiment was repeated.

\section{Effect of diffusible communication signals on antimicrobial activity}

The effect of DCSs on AZ78 antimicrobial activity was evaluated on Rhizosphere Mimicking Agar (RMA) [33] and the experimental design was made up to 8 treatments (Table S2). Inhibitory activity of AZ78 against $P$. ultimum was evaluated by using the classic dual-culture method. In brief, $10 \mu \mathrm{L}$ of AZ78 cell suspension $\left(1 \times 10^{8} \mathrm{CFU} / \mathrm{mL}\right)$ were spot-inoculated at $3 \mathrm{~cm}$ of the edge of a plate. After $48 \mathrm{~h}$ incubation at $27^{\circ} \mathrm{C}$, mycelium plugs $(4 \mathrm{~mm})$ were cut from the edge of one-week old $P$. ultimum plate, placed at $2.5 \mathrm{~cm}$ distance from AZ78 and incubated at $25^{\circ} \mathrm{C}$ for $168 \mathrm{~h}$. AZ78 activity against Rhodococcus fascians LMG 3605 was determined by spot-inoculating $10 \mu$ l of AZ78 cell suspension $\left(1 \times 10^{8} \mathrm{CFU} / \mathrm{mL}\right)$ in the centre of a RMA plate. After $48 \mathrm{~h}$ incubation at $27^{\circ} \mathrm{C}, \mathrm{AZ78}$ cells were killed by exposure to chloroform vapour for $60 \mathrm{~min}$ [34]. Dishes were aerated under a laminar flow for 60 min, overlaid with $4 \mathrm{~mL}$ of $0.45 \%$ agar PBS containing $R$. fascians $\mathrm{LMG} 3605\left(1 \times 10^{7} \mathrm{CFU} / \mathrm{mL}\right)$ and incubated at $27^{\circ} \mathrm{C}$ for $72 \mathrm{~h}$. RMA dishes seeded only with $P$. ultimum or $R$. fascians LMG 3605 were used as control. 
124 Pictures were obtained with Bio-Rad Quantity One software implemented in a Bio-Rad GelDoc 125 Imaging system (Bio-Rad Laboratories, Hercules, California, USA). Inhibitory activity was 126 quantified by scoring P. ultimum or R. fascians LMG 3605 growth area $\left(\mathrm{cm}^{2}\right)$ using Image $1.52 \mathrm{a}$ 127 [35] and calculated according to the formulas below:

Antioomycete activity $(\%)=\left(\frac{\text { Inhibition of mycelial growth in presence of DCS }}{\text { Inhibition of mycelial growth in absence of DCS }}-1\right) \times 100$ where

Inhibition of mycelial growth $=\left(1-\frac{\text { Mycelium area in presence of } \text { L.capsici AZ78 }}{\text { Mycelium area in absence of } \text { L.capsici AZ78 }}\right) \times 100$

The effect of DCSs on AZ78 antibacterial activity was assessed as follows:

Antibacterial activity $(\%)=\left(\frac{\text { Inhibition zone area in presence of DCS }}{\text { Inhibition zone area in absence of DCS }}-1\right) \times 100$

In both cases, treatments included five replicates and experiments were repeated.

The AZ78 response to DCSs was evaluated on RMA and the experimental design was made up to 8 treatments in triplicate (Table S2). Ten microliters of AZ78 cell suspension $\left(1 \times 10^{10} \mathrm{CFU} / \mathrm{mL}\right)$ were spot-inoculated in the centre of a RMA plate and incubated at $27^{\circ} \mathrm{C}$ for $48 \mathrm{~h}$. Plugs $(7 \mathrm{~mm}$ diameter) were collected from the AZ78 macrocolonies, immediately frozen in liquid nitrogen and stored at $80^{\circ} \mathrm{C}$. Frozen samples were processed according to Brescia et al., [33] and total RNA was extracted using Spectrum Plant Total RNA Kit (Sigma-Aldrich). DNase treatment was performed with the RNase-Free DNase set (Qiagen, Hilden, Germany). RNA integrity and concentration were assessed using a 2200 TapeStation System (Agilent Technologies, Santa Clara, California, USA) and a Qubit (ThermoFisher Scientific), respectively. 
146 Library construction and Illumina Sequencing were carried out at Fasteris (Plan-les-Ouates,

147 Switzerland). Ribosomal RNA (rRNA) depletion was performed using the Ribo-Zero rRNA Removal 148 Kits (Bacteria) (Illumina, San Diego, California, USA). Complementary DNA (cDNA) libraries were 149 synthesised using TruSeq Stranded mRNA Library Prep (Illumina, USA), they were multiplexed (two 150 libraries per lane) and paired-end reads of 150 nucleotides were obtained using an Illumina HiSeq 1514000 (Illumina), resulting in $\sim 7-42$ million reads per sample (Table S3). Raw sequences were 152 deposited at the Sequence Read Archive of the NCBI under BioProject number PRJNA714393.

153 Sequence analysis was carried out using Omicsbox 1.3.11 (www.biobam.com/omicsbox). Illumina 154 HiSeq data was assessed for quality using FastQC [36]. Raw reads for each sample were trimmed to 155 increase overall quality using Trimmomatic 0.38 [37]. The resulting reads were aligned to AZ78 156 genome (Table S1) using the STAR 2.7.5a [38] and read counts were extracted from STAR 157 alignments using HTSeq [39].

\section{Identification of differentially expressed genes and functional annotation of RNA-Seq}

Genes with zero counts in all replicates were excluded from the analysis and raw counts were normalised using the trimmed mean of M-values method [40]. Differentially expressed genes (DEGs) were identified using edgeR 3.28.0 [41] using a p-value $<0.01$ and a log fold change (FC) of at least 1-fold upregulation/downregulation as cut off values. Venn diagrams summarizing DEGs distribution were drawn with VennPainter [42]. Hierarchical clustering and heat maps were created with TreView3 [43].

The protein sequences of all predicted genes [34] were functionally annotated using Blast2Go (http://www.blast2go.org) [44]. Default settings were applied and a minimum E-value of $10^{-5}$ was imposed as cut off. DEGs were further annotated based on the NCBI gene description and classified in 20 functional categories. 


\section{Results}

First-strand cDNA was synthetized from $600 \mathrm{ng}$ of purified RNA with SuperScript III Reverse Transcriptase (Invitrogen, Carlsbad, California, USA) using random hexamers, according to manufacturer's instructions. qRT-PCR reactions were carried out with Platinium SYBR Green qPCR Super-Mix-UDG (Invitrogen, USA) and specific primers (Table S4) were designed using Primer3 software [45]. Primer specificity was assessed using PCR before gene expression analysis. qRT-PCR reactions were run for 50 cycles $\left(95{ }^{\circ} \mathrm{C}\right.$ for $15 \mathrm{~s}$ and $60{ }^{\circ} \mathrm{C}$ for $45 \mathrm{~s}$ ) on a LightCycler 480 (Roche Diagnostics, Mannheim, Germany). Each sample was examined in three technical replicates and dissociation curves were analysed to verify the specificity of each amplification reaction. LightCycler 480 software, version 1.5 (Roche Diagnostics, Mannheim, Germany) was used to extract cycle threshold $(\mathrm{Ct})$ values based on the second derivative calculation and the LinReg software, version 11.0, was used to calculate reaction efficiencies for each primer pair [46]. Relative expression levels were calculated according to Pfaffl equation [47] using AZ78 growing in RMA as calibrator. The housekeeping gene recA (AZ78_1089; [34]) was used as constitutive gene for normalization. The linear relationship between the RNA-Seq $\log _{2}$ FC values and the qRT-PCR $\log _{2}$ FC values of selected genes was estimated by Pearson correlation analysis.

\section{Statistical analysis}

Percentage values were arcsine square root transformed to normalize distributions and to equalize variances. Comparisons between repeated experiments of antimicrobial activity were done using twoway analysis of variance (ANOVA) and the data were pooled when no significant differences were found according to the $F$-test $(p>0.05)$. Data were analysed using one-way ANOVA and Tukey’s test $(\alpha=0.05)$ was used to detect significant differences. Statistical analyses were carried out using IBM SPSS Statistics for Windows, Version 21.0 (IBM Corp, Armonk, New York, USA).

\section{Cell-cell communication systems in Lysobacter capsici AZ78 genome}


194 Putative genes encoding the DSF response regulator RpfG (AZ78_0630) and the turn-over signal

195

196

197

198

199

200

201

202

203

204

205

206

207

208

209

210

211

212

213

214 RpfB (AZ78_0629) were found in the AZ78 genome and showed an identity greater than 80\% (at amino acid level) with its homologues in L. enzymogenes C3, Sm K279a, and Xcc ATCC 33913 (Table 1). rpfG and $r p f B$ genes in AZ78 were closely related to their homologues in other Lysobacter spp. and clustered together (Figure S1). AZ78 genome also included a putative DSF sensor RpfC (AZ78_3298) and a crotonase/enoyl-Coenzyme A (RpfF) (AZ78_3297) with low similarity compared to L. enzymogenes C3, Sm K279a, and Xcc ATCC $33913^{\mathrm{T}}$ (Table 1, Figure S2). The $r p f F / r p f C$ region $(3,947,548$ - 3,948,450 bp) was located far from the $r p f G / r p f B$ region $(857,114-$ 859,152 bp) in AZ78 (Figure 1a). DSF biosynthesis was confirmed by AZ78 ability to induce the glucuronidase activity in Xcc 8523 pL6engGUS like the control strain Xcc 8004 (Figure 1b).

As for VOCs, a putative gene encoding IND synthase (AZ78_4108) was found in AZ78 genome. Homologues of qseB (AZ78_2946) and qseC (AZ78_2945) involved in IND regulation were also found in AZ78 genome and showed high identity with L. enymogenes C3 homologue (Table 1). Homologues of IND synthase and QseB/QseC system were also present in other Lysobacter spp. (Figure S3).

A gene encoding a putative chorismatase needed for DFs production was found in AZ78 genome (AZ78_3466) with an identity of 64\% at amino acid level with the homologue xanB2 (XCC4014) of Xcc ATCC $33913^{\mathrm{T}}$. Furthermore, a homologue of the LysR family transcription factor involved in the DF regulatory cascade, was identified in AZ78 genome (AZ78_2901) (Table 1). Chorismatase and LysR transcription factor were also typical among other Lysobacter spp. (Figure S4).

AZ78 luxR gene (AZ78_4823), responsible for the detection and response to AHLs, had an amino acid identity of 77 and 48 with the homologues of L. enzymogenes C3 and Sm K279a, respectively (Table 1). luxR phylogenetic analysis showed that AZ78 luxR homolog clustered together with other members of L. capsici (Figure S4). LuxR was not associated to its cognate AHL synthase (LuxI) in AZ78 or in other Lysobacter species, with only exception of L. daejeonensis GH1-9 ${ }^{\mathrm{T}}$ having a luxI 
219 homologue. The absence of LuxI homologs was confirmed by the inability of AZ78 to restore $\beta$ 220 galactosidase activity and violacein production in the reporter strains A. tumefaciens NT1 pZRL4 and 221 C. violaceum CV026, respectively (Figure S5). In contrast, L. daejeonensis GH1-9 ${ }^{\mathrm{T}}$ was able to restore violacein production in C. violaceum $\mathrm{CV} 026$ confirming relation between the presence of luxI and AHL production.

Diffusible communication signals affect Lysobacter capsici AZ78 growth and antimicrobial activity

GLY, BUT, 3HBA, 4HBA and AHL had no effect on AZ78 growth curves, whereas LeDSF3 and IND slowed down AZ78 growth compared to the untreated control (Figure S6).

Exogenous addition of DCSs to RMA showed no effect on P. ultimum and $R$. fascians LMG 3605 growth, but it modulated AZ78 antimicrobial activity against $P$. ultimum and $R$. fascians LMG 3605 (Figure 2). LeDSF3, 4HBA, and IND decreased AZ78 inhibitory activity against P. ultimum by a 5, 22 and $47 \%$, respectively. In contrast GLY, BUT, 3HBA, and AHL increased AZ78 inhibitory activity against $P$. ultimum up to a 7\% (Figure 2). LeDSF3, IND, 3HBA, 4HBA and AHL decreased AZ78 inhibitory activity against $R$. fascians LMG 3605 up to a $31 \%$, while BUT and GLY increased AZ78 antibacterial activity by a 9 and $48 \%$ respectively (Figure 2). Changes were particularly relevant for IND and GLY.

\section{Transcriptional response of Lysobacter capsici AZ78 to diffusible communication signals}

The expression of $21 \%$ of all AZ78 genes was significantly affected by DCSs $\left(\left|\log _{2} \mathrm{FC}\right|>1\right.$ and $p<$ 0.01). The largest number of DEGs, 636 genes (about 11.9\% of AZ78 transcriptome), was found upon exposure of AZ78 to LeDSF3 (Table 2). This was followed by IND (603 DEGs, $11.3 \%$ of total genes), GLY (292 DEGs, 5.5\% of total genes), BUT (237 DEGs, 4.4\% of total genes), 3HBA (101 DEGs, $1.9 \%$ of total genes), and 4BHA (58 DEGs, $1.1 \%$ of total genes). The lowest number of DEGs (24, $0.5 \%$ of total genes) occurred among cells treated with AHL (Table 2). RNA-Seq results were 
validated by the relative expression level of 10 selected genes assessed using qRT-PCR (Table S4).

A close correlation (Pearson's $r=0.95$ ) was observed between $\log _{2}$ FC measured with RNA-Seq and qRT-PCR (Figure S7).

Venn diagrams (Figure S8) revealed overlaps among the seven conditions, but it did not identified genes modulated by all seven DCSs. Moreover, a heatmap (Figure 3) showed that GLY and BUT clustered together, likewise 3HBA, 4HBA, and AHL. Instead, LeDSF3 and IND grouped independently.

\section{The active response of Lysobacter capsici AZ78 to diffusible communication signals}

Functional annotation of AZ78 genes modulated by DCSs revealed that up-regulated DEGs were mainly related to global metabolism, growth, RNA transcription and degradation; transport, phosphotransferase systems, and secretion (Figure 4). Conversely, down-regulated DEGs were mainly related to DNA metabolism.

LeDSF3 regulated a total of 68 genes involved in transport, phosphotransferase systems, and secretion; 51 genes involved in global metabolism; 35 genes involved in RNA transcription and degradation; 35 genes involved in translation, 34 genes involved growth, and 34 genes involved in amino acid metabolism (Figure 4, Table S5). The category of transport, phosphotransferase systems, and secretion was affected by IND (55 genes) (Figure 4, Table S6), followed by global metabolism (54 genes), RNA transcription and degradation (41 genes), growth (40 genes), and antagonism (34 genes). GLY, BUT, and 3HBA modulated a relevant number of genes related to global metabolism and transport, phosphotransferase systems, and secretion (Figure 4, Tables S7, S8, S9). Moreover, GLY and BUT modulated a relevant number of genes classified into RNA transcription and degradation and translation, among which tRNA genes were mainly down-regulated. Genes related 
Efflux pumps, type IV pilus biogenesis, antagonism, cell-cell communication systems, and secretion systems are influenced by diffusible communication signals in Lysobacter capsici AZ78

Many genes ascribed to transport, phosphotransferase systems, and secretion were involved in drug and metal (particularly iron) transport (Tables S5-S11). Major Facilitator Superfamily (MFS) transporters, like the multidrug exporter gene yceL (AZ78_2682), were up-regulated by LeDSF3 (Figure 5). Resistance-Nodulation-Division (RND) efflux system genes were up-regulated by DCSs, especially by AHL (Figure 5, Table S11). TonB-dependent receptors involved in the uptake of ironsiderophore complexes or vitamins were up-regulated by LeDSF3 and down-regulated by IND (Tables S5, S6). Additionally, DCSs up-regulated a relevant set of transcription regulators belonging to the AraC, ArsR, TetR, MerR and MarR families (Tables S5-S11).

With the only exception of AHL, DCSs modulated the expression of genes involved in type IV pilus (T4P) biosynthesis. LeDSF3 up-regulated the expression of pilW1-pilV1-fimU1 genes (AZ78_1459AZ78_1461), related to minor pilins; pilR from two-component system pilR-pilS (AZ78_4278AZ78_4279); and pilH (AZ78_4388), pilI (AZ78_4389), chpA (AZ78_4391), chpB (AZ78_4392) and $c p h C$ (AZ78_4393) from the pilus-specific chemotaxis system (Pil-Chp) (Figure 5, Table S5). Genes encoding minor pilins, pilRS, and Pil-Chp were also up-regulated in GLY, BUT, and 4HBA (Figure 5; Tables S7, S8, S10). IND up-regulated the expression of fimV (AZ78_0130), pilV1, pilRpilS, and pilH, while it down-regulated pilF (AZ78_0791), pilO and pilM (AZ78_2052 and AZ78_2054), and the major pilin pilA (AZ78_4276-4277) (Figure 5, Table S6). LeDSF3 and IND down-regulated a relevant number of involved in antagonism. IND down-regulated the biosynthetic gene cluster AZ78_1095-AZ78_1102, responsible for the production of HSAF [33] (Figure 5; Table S6), and several extracellular proteases (e.g. AZ78_2802 and AZ78_3223) (Table S6). Moreover, IND up-regulated a diguanylate cyclase harbouring a GGDEF motif (AZ78_4062) possibly related to cyclic-di-GMP (c-di-GMP) biosynthesis (Table S6). Other genes related to antagonism, such as 
genes encoding Rhs toxins and RebB proteins, responsible for the expression of killing traits, were down-regulated by LeDSF3 and IND (Figure 5; Tables S5, S6).

DCS also caused changes in the expression of genes involved in the reception and regulation of DCSs in AZ78 (Figure 5). For instance, the transcription factor LysR (AZ78_2901) was up-regulated by IND and BUT, qseB (AZ78_2946) was up-regulated by AHL and luxR (AZ78_4823) by IND.

Genes related to Type I secretion system (T1SS) were mostly up-regulated by all DCSs, especially by LeDSF3 and IND (Figure 5; Tables S5, S6). As an example, the ABC transporter yadG (AZ78_2378) was up-regulated by LeDSF3, IND, and GLY. Likewise, lapE gene (AZ78_3791), responsible for the secretion of the RTX adhesin LapA, was up-regulated by LeDSF3, IND, GLY, BUT and 4HBA. Type II secretion system (T2SS) genes, such as gspG (AZ78_4200) and gspH (AZ78_4201), were up-regulated by LeDSF3, IND, GLY, and BUT (Figure 5; Tables S5-S8). On the contrary, the expression of Type III secretion system (T3SS) was down-regulated by GLY and 3HBA (Figure 5; Tables S7, S9). Genes associated with type IV secretion system (T4SS), such as the duplicate gene pair located through the genome virB6 (AZ78_0512) was down-regulated by LeDSF3, IND and GLY (Figure 5; Tables S5-S7). Additionally, IND repressed virB5b (AZ78_0703) and virB3 (AZ78_0706), from the virB1-virB11 complex, and the duplicates gene pairs located through the genome virB5b (AZ78_4957) and virB6 (AZ78_0514 and AZ78_4958). ShlB from the two-partner secretion of Type V secretion system (T5bSS) was down-regulated by BUT and 4HBA (Figure 5; Tables S8, S10). Finally, DCSs regulated general secretory (Sec) pathways, such as $\sec B$ (AZ78_2696) up-regulated by IND and BUT (Tables S6, S8), or secF (AZ78_4807) down-regulated by LeDSF3 and BUT (Figure 5; Tables S6, S8).

\section{Discussion}

The behaviour of bacterial species mainly relies on communication systems [2] and many secreted metabolites characterising the cooperation among microorganisms, as well as antibiotics and toxins 
involved in microbial competition, are controlled by DCSs [48-50]. DCSs are not only involved in signalling among self-cells, but also in the detection of specific cues produced by other strains or species [48]. In fact, many bacterial species have receptors for DCSs that are not produced by the same species, such as LuxR solos, and abundant two-component signalling systems [48]. Genome mining results indicate that AZ78 and Lysobacter spp. may (at least) produce DSFs, IND, DFs and perceive DSFs, IND, DFs, and AHLs. As a consequence, DCSs (mainly IND and GLY) influenced AZ78 antibacterial and antioomycetes activity in vitro. Different intra-, interspecies and interkingdom DCSs might be used as cues for AZ78 to favour the regulation of molecular pathways related to cell persistence in the rhizosphere or for coercion (Figure 6). Thus, transcriptome profiles showed that DCSs might contribute to alert AZ78 against toxic compounds in the rhizosphere by triggering the expression of genes encoding efflux pumps that could actively extrude antibiotics, heavy metals, biocides, and solvents [51]. In addition, DCSs might help cells to escape from adverse conditions or to reach nutrients [52]. For example, LeDSF3, GLY, and BUT up-regulated the expression of genes related to the biogenesis of T4P involved in twitching motility. T4P-driven twitching motility is involved in a variety of physiological and social behaviours of a wide range of bacteria [53, 54]. For instance, twitching motility is required for colonization and infection of phytopathogenic fungi and oomycetes in Lysobacter spp. [55, 56] and it seems to be a DSF-dependent trait in L. brunescens and L. enzymogenes $[9,20,21]$. Interestingly, up-regulation of T4P by GLY and BUT came along with increased antimicrobial activity, suggesting that AZ78 might colonize new niches and form a stable community to outcompete other (micro)organisms upon the perception of these DCSs. Moreover, GLY and BUT down-regulated the transcription of tRNA genes. In E. coli down-regulation of tRNA was associated with the ability of this bacterium to cope with amino acid starvation (reviewed in [57]) and oxidative stress [58], which prompted us to think that GLY and BUT might enhance the stress response in AZ78 to promote survival and adaptation in the rhizosphere. In contrast, IND caused a dysregulation of T4P genes in AZ78 with possible losses of T4P functionality. Accordingly, IND decreases motility and biofilm formation in E. coli [59-62], probably as a manner to save energy and 
regulate growth dynamics [63]. In support to this hypothesis, IND diminished the AZ78 cell growth, although it was not possible to formulate a clear conclusion as knowledge about IND functions is contrasting [64-66].

In addition to escape mechanisms, Lysobacter cells might outcompete other microorganisms by producing extracellular lytic enzymes and antibiotics. Previous findings showed that HSAF biosynthesis is positively regulated by DSF, IND and 4HBA in Lysobacter spp. [9, 19, 21]. Yet, in AZ78 IND reduced antioomycete activity and down-regulated the expression of the HSAF biosynthetic gene cluster. The down-regulation of HSAF related genes by IND might be associated to the simultaneous up-regulation of the solo LuxR solo (AZ78_4823), as previously reported in $L$. enzymogenes $\mathrm{OH} 11$, where overexpression of lesR (LuxR homologue) leads to a decrease in HSAF production [67, 68]. Moreover, IND up-regulated several transcription regulators (among which various tetR repressors [69], like AZ78_0770 and AZ78 3232) that might be involved in HSAF biosynthesis regulation in AZ78, as found for LetR (a TetR-family protein) in L. enzymogenes OH11 [70]. The expression of HSAF biosynthetic cluster is also negatively regulated by cyclic-di-GMP (cdi-GMP) in L. enzymogenes OH11 [52]. Interestingly, IND up-regulated the expression of a diguanylate cyclase (AZ78_4062) that might be involved in c-di-GMP biosynthesis, implying a regulation role of c-di-GMP in HSAF production in AZ78. Besides producing secondary metabolites with antimicrobial activity, AZ78 might produce diffusible proteinaceous toxins and toxins deployed by contact-dependent systems, such as Rhs toxins, which mediate growth inhibition of neighbouring cells in Dickeya dadantii [71], or R-bodies, which are responsible for cell membrane disruption and toxins delivery in several bacterial genera $[72,73]$. Thus, the down-regulation of several $r h s$ and $r e b$ genes required for Rhs toxins and R-bodies synthesis by LeDSF3 and IND might have contributed to lower AZ78 the antioomycete and antibacterial activity. Moreover, AZ78 down-regulated signal transduction pathways in presence of IND, such as TonB-dependent receptors, which play a key role in microbial competition with the uptake of iron-siderophore complex or vitamins [74]. 
Bacteria often use secretion systems to manipulate and kill rival bacterial and eukaryotic cells [75, 76]. Of those, T3SS, T4SS and T6SS are related to the establishment of pathogenic interactions with microbial eukaryotic hosts in Lysobacter spp. [77]. Thus, modulation of genes related to secretion systems might result in gain/loss of ability to compete with other (micro)organisms [78]. In agreement with this statement, IND down-regulated T4SS and decreased antimicrobial activity in AZ78. Downregulation of T4SS by IND might be related to the overexpression of diguanylate cyclases (e.g., AZ78_4062), responsible for c-di-GMP increase and T4SS inactivation in A. tumefaciens [79]. However, T3SS was down-regulated by GLY and 3HBA with no decrease in AZ78 toxic activity, suggesting that it was probably repressed to save energy under conditions where it does not provide an advantage, as found in P. aeruginosa [80], Vibrio harveyi [81] and Yersinia pseudotuberculosis [82].

Overall, functional and transcriptome analysis of AZ78 in presence of signalling communication systems clarified its behaviour in the rhizosphere. Our results show that GLY and BUT might facilitate rhizosphere competence of Lysobacter spp. and soil disease suppressiveness to plant pathogens. On the other hand, IND might prevent Lysobacter spp. from growing at high cell densities and decrease motility. Moreover, IND and LeDSF3 might decrease Lysobacter spp. ability to control phytopathogenic microorganisms. Manipulating DCSs levels in the rhizosphere could therefore provide efficient means to favour the establishment and functioning of beneficial bacteria, such as Lysobacter strains, at the root-soil interface.

\section{Acknowledgements}

This work was founded by the European Union's Horizon 2020 research and innovation programme under the Marie Skłodowska-Curie grant agreements no. 797028.

\section{Author contributions}


$\mathrm{AB}$ and GP conceived the study, performed the experiments, analysed the data, conceptualized, and drafted the manuscript. MP helped in the experimental set up and provided input and proof reading of the manuscript. IP provided input and proof reading of the manuscript.

\section{Conflict of interest}

The authors declare that they have no conflict of interest.

\section{References}

1. Raaijmakers JM, Paulitz TC, Steinberg C, Alabouvette C, Moënne-Loccoz Y. The rhizosphere: a playground and battlefield for soilborne pathogens and beneficial microorganisms. Plant Soil

2. Venturi V, Keel C. Signaling in the rhizosphere. Trends Plant Sci 2016; 21: 187-198.

3. Case RJ, Labbate M, Kjelleberg S. AHL-driven quorum-sensing circuits: their frequency and

4. Papenfort K, Bassler BL. Quorum sensing signal-response systems in Gram-negative bacteria.

5. Stephens K, Bentley WE. Synthetic Biology for Manipulating Quorum Sensing in Microbial

6. Barber CE, Tang JL, Feng JX, Pan MQ. A novel regulatory system required for pathogenicity

7. Ryan RP, An S qi, Allan JH, McCarthy Y, Dow JM. The DSF Family of Cell-Cell Signals: An Expanding Class of Bacterial Virulence Regulators. PLoS Pathog 2015; 11: 1-14. 
8. He Y-W, Wu J, Zhou L, Yang F, He Y-Q, Jiang B-L, et al. Xanthomonas campestris Diffusible Factor Is 3-hydroxybenzoic acid and is associated with Xanthomonadin biosynthesis, cell viability, antioxidant activity, and systemic invasion. Mol Plant-Microbe Interact 2011; 24: 948-957.

9. Qian G, Wang Y, Liu Y, Xu F, He YW, Du L, et al. Lysobacter enzymogenes uses two distinct cell-cell signaling systems for differential regulation of secondary-metabolite biosynthesis and colony morphology. Appl Environ Microbiol 2013; 79: 6604-6616.

10. Zhou L, Huang T, Wang J, Sun S, Chen G, Poplawsky A, et al. The Rice Bacterial Pathogen Acid via XanB2 for Use in Xanthomonadin, Ubiquinone, and Exopolysaccharide Biosynthesis. Mol Plant-Microbe Interact 2013; 26: 1239-1248.

11. Han Y, Wang Y, Tombosa S, Wright S, Huffman J, Yuen G, et al. Identification of a small molecule signaling factor that regulates the biosynthesis of the antifungal polycyclic tetramate macrolactam HSAF in Lysobacter enzymogenes. Appl Microbiol Biotechnol 2015; 99: 801-

12. Schmidt R, Cordovez V, de Boer W, Raaijmakers J, Garbeva P. Volatile affairs in microbial interactions. ISME J 2015; 9: 2329-2335.

13. Lee JH, Lee J. Indole as an intercellular signal in microbial communities. FEMS Microbiol $\operatorname{Rev} 2010 ; 34:$ 426-444.

14. Kim K, Lee S, Ryu C-M. Interspecific bacterial sensing through airborne signals modulates locomotion and drug resistance. Nat Commun 2013; 4: 1809.

15. Puopolo G, Tomada S, Pertot I. The impact of the omics era on the knowledge and use of Lysobacter species to control phytopathogenic micro-organisms. J Appl Microbiol 2017; 124: 
16. Li S, Jochum CC, Yu F, Zaleta-Rivera K, Du L, Harris SD, et al. An antibiotic complex from Lysobacter enzymogenes strain C3: antimicrobial activity and role in plant disease control. Phytopathology 2008; 98: 695-701.

17. Yuen GY, Broderick KC, Jochum CC, Chen CJ, Caswell-Chen EP. Control of cyst nematodes by Lysobacter enzymogenes strain $\mathrm{C} 3$ and the role of the antibiotic HSAF in the biological

18. Su Z, Chen H, Wang P, Tombosa S, Du L, Han Y. 4-Hydroxybenzoic acid is a diffusible factor that connects metabolic shikimate pathway to the biosynthesis of a unique antifungal

19. Han Y, Wang Y, Yu Y, Chen H, Shen Y, Du L. Indole-Induced Reversion of Intrinsic 444 Multiantibiotic Resistance in Lysobacter enzymogenes. Appl Environ Microbiol 2017; 83: 114.

20. Feng T, Han Y, Li B, Li Z, Yu Y, Sun Q, et al. Interspecies and intraspecies signals synergistically regulate Lysobacter enzymogenes twitching motility. Appl Environ Microbiol

21. Ling J, Zhu R, Laborda P, Jiang T, Jia Y, Zhao Y, et al. LbDSF, the Lysobacter brunescens quorum-sensing system diffusible signaling factor, regulates anti-xanthomonas XSAC biosynthesis, colony morphology, and surface motility. Front Microbiol 2019; 10: 1-14.

22. Aziz RK, Bartels D, Best AA, DeJongh M, Disz T, Edwards RA, et al. The RAST Server: rapid annotations using subsystems technology. BMC Genomics 2008; 9: 75. 
24. Thompson JD, Higgins DG, Gibson TJ. CLUSTAL W: improving the sensitivity of progressive multiple sequence alignment through sequence weighting, position-specific gap penalties and weight matrix choice. Nucleic Acids Res 1994; 22: 4673-4680.

25. Kimura M. The Neutral Theory and Molecular Evolution BT - My Thoughts on Biological Evolution. In: Kimura M (ed).2020. Springer Singapore, Singapore, pp 119-138.

26. Saitou N, Nei M. The neighbor-joining method: a new method for reconstructing phylogenetic trees. Mol Biol Evol 1987; 4: 406-425.

27. Kumar S, Stecher G, Tamura K. MEGA7 : Molecular Evolutionary Genetics Analysis Version 7.0 for Bigger Datasets Brief communication. Mol Biol Evol 2016; 33: 1870-1874.

28. Felsenstein J. Confidence Limits on Phylogenies: An Approach Using the Bootstrap. Evolution (N Y) 1985; 39: 783-791.

29. Steindler L, Venturi V. Detection of quorum-sensing N-acyl homoserine lactone signal molecules by bacterial biosensors. FEMS Microbiol Lett 2007; 266: 1-9.

30. Cha C, Gao P, Chen YC, Shaw PD, Farrand SK. Production of acyl-homoserine lactone quorum-sensing signals by gram-negative plant-associated bacteria. Mol Plant Microbe Interact 1998; 11: 1119-1129.

31. Slater H, Alvarez-morales A, Barber CE, Daniels MJ, Dow JM, Christine E, et al. A twocomponent system involving an HD-GYP domain protein links cell-cell signalling to pathogenicity gene expression in Xanthomonas campestris. Mol Microbiol 2000; 38: 9861003.

32. Puopolo G, Aida R, Pierson LS, Zoina A. Selection of a new Pseudomonas chlororaphis strain for the biological control of Fusarium oxysporum f. sp. radicis-lycopersici. Phytopathol Mediterr 2011; 50: 228-235. 
33. Brescia F, Marchetti-Deschmann M, Musetti R, Perazzolli M, Pertot I, Puopolo G. The rhizosphere signature on the cell motility, biofilm formation and secondary metabolite production of a plant-associated Lysobacter strain. Microbiol Res 2020; 234: 126424.

34. Puopolo G, Tomada S, Sonego P, Moretto M, Engelen K, Perazzolli M, et al. The Lysobacter capsici AZ78 genome has a gene pool enabling it to interact successfully with phytopathogenic microorganisms and environmental factors. Front Microbiol 2016; 7: 1-14.

35. Schneider CA, Rasband WS, Eliceiri KW. NIH Image to ImageJ: 25 years of image analysis. Nat Methods 2012; 9: 671-675.

36. Andrews S. FASTQC. A quality control tool for high throughput sequence data (online). 2010.

37. Bolger AM, Lohse M, Usadel B. Trimmomatic: a flexible trimmer for Illumina sequence data. Bioinformatics 2014; 30: 2114-2120.

38. Dobin A, Davis CA, Schlesinger F, Drenkow J, Zaleski C, Jha S, et al. STAR: ultrafast universal RNA-seq aligner. Bioinformatics 2013; 29: 15-21.

39. Anders S, Pyl PT, Huber W. HTSeq - a Python framework to work with high-throughput sequencing data. Bioinformatics 2015; 31: 166-169.

40. Robinson MD, Oshlack A. A scaling normalization method for differential expression analysis of RNA-seq data. Genome Biol 2010; 11: R25.

41. Robinson MD, McCarthy DJ, Smyth GK. edgeR: a Bioconductor package for differential expression analysis of digital gene expression data. Bioinformatics 2010; 26: 139-140.

42. Lin G, Chai J, Yuan S, Mai C, Cai L, Murphy RW, et al. VennPainter: A Tool for the Comparison and Identification of Candidate Genes Based on Venn Diagrams. PLoS One 2016; 11: $1-12$. 
501 43. Saldanha AJ. Java Treeview-extensible visualization of microarray data. Bioinformatics 2004; 20: 3246-3248.

44. Conesa A, Götz S, García-Gómez JM, Terol J, Talón M, Robles M. Blast2GO: a universal tool for annotation, visualization and analysis in functional genomics research. Bioinformatics 2005; 21: 3674-3676.

45. Untergasser A, Cutcutache I, Koressaar T, Ye J, Faircloth BC, Remm M, et al. Primer3 — new capabilities and interfaces. Nucleic Acids Res 2012; 40: e115.

46. Ruijter JM, Ramakers C, Hoogaars WMH, Karlen Y, Bakker O, Hoff MJB Van Den, et al. Amplification efficiency : linking baseline and bias in the analysis of quantitative PCR data.

47. Pfaffl MW. A new mathematical model for relative quantification in real-time RT-PCR. Nucleic Acids Res 2001; 29: e45.

48. Cornforth DM, Foster KR. Competition sensing: the social side of bacterial stress responses. Nat Rev Microbiol 2013; 11: 285-93.

49. Hibbing ME, Fuqua C, Parsek MR, Peterson SB. Bacterial competition: surviving and thriving in the micorbial jungle. Natl Rev Microbiol 2010; 8: 15-25.

50. Schuster M, Joseph Sexton D, Diggle SP, Peter Greenberg E. Acyl-Homoserine Lactone Quorum Sensing: From Evolution to Application. Annu Rev Microbiol 2013; 67: 43-63.

51. Blanco P, Hernando-Amado S, Reales-Calderon J, Corona F, Lira F, Alcalde-Rico M, et al. Bacterial Multidrug Efflux Pumps: Much More Than Antibiotic Resistance Determinants. Microorganisms 2016; 4: 14. 
Type IV Pilus Synthesis, Controls Antifungal Antibiotic Production via a Cyclic di-GMP Pathway. Appl Environ Microbiol 2017; 83: 1-19.

53. Zhang Y, Ducret A, Shaevitz J, Mignot T. From individual cell motility to collective behaviors: insights from a prokaryote, Myxococcus xanthus. FEMS Microbiol Rev 2012; 36: 149-164.

54. Burrows LL. Pseudomonas aeruginosa Twitching Motility: Type IV Pili in Action. Annu Rev Microbiol 2012; 66: 493-520.

55. Patel N, Cornejo M, Lambert D, Craig A, Hilman B, Kobayashi D. A multifunctional role for the type IV pilus in the bacterial biological control agent Lysobacter enzymogenes. Phytopathology. 2011. p S138.

56. Tomada S, Sonego P, Moretto M, Engelen K, Pertot I, Perazzolli M, et al. Dual RNA-Seq of Lysobacter capsici AZ78 - Phytophthora infestans interaction shows the implementation of attack strategies by the bacterium and unsuccessful oomycete defense responses. Environ Microbiol 2017; 19: 4113-4125.

57. Potrykus K, Cashel M. (p)ppGpp: Still Magical? Annu Rev Microbiol 2008; 62: 35-51.

58. Zhong J, Xiao C, Gu W, Du G, Sun X, He Q-Y, et al. Transfer RNAs Mediate the Rapid Adaptation of Escherichia coli to Oxidative Stress. PLOS Genet 2015; 11: e1005302.

59. Domka J, Lee J, Wood TK. YliH (BssR) and YceP (BssS) regulate Escherichia coli K-12 biofilm formation by influencing cell signaling. Appl Environ Microbiol 2006; 72: 2449-2459.

60. Bansal T, Englert D, Lee J, Hegde M, Wood TK, Jayaraman A. Differential effects of epinephrine, norepinephrine, and indole on Escherichia coli O157:H7 chemotaxis, colonization, and gene expression. Infect Immun 2007; 75: 4597-4607.

61. Mufti R, Amna, Rafique M, Haq F, Munis MFH, Masood S, et al. Genetic diversity and metal 

resistance assessment of endophytes isolated from Oxalis corniculata. Soil Environ 2015; 34: 89-99.

62. Lee J, Zhang X-S, Hegde M, Bentley WE, Jayaraman A, Wood TK. Indole cell signaling occurs primarily at low temperatures in Escherichia coli. ISME J 2008; 2: 1007-1023.

63. Nadell CD, Xavier JB, Levin SA, Foster KR. The Evolution of Quorum Sensing in Bacterial Biofilms. PLOS Biol 2008; 6: e14.

64. Lee J, Attila C, Cirillo SLG, Cirillo JD, Wood TK. Indole and 7-hydroxyindole diminish Pseudomonas aeruginosa virulence. Microb Biotechnol 2009; 2: 75-90.

65. Mueller RS, Beyhan S, Saini SG, Yildiz FH, Bartlett DH. Indole acts as an extracellular cue regulating gene expression in Vibrio cholerae. J Bacteriol 2009; 191: 3504-3516.

66. Mueller RS, McDougald D, Cusumano D, Sodhi N, Kjelleberg S, Azam F, et al. Vibrio cholerae strains possess multiple strategies for abiotic and biotic surface colonization. $J$ Bacteriol 2007; 189: 5348-5360.

67. Qian G, Xu F, Venturi V, Du L, Liu F. Roles of a solo LuxR in the biological control agent Lysobacter enzymogenes strain OH11. Phytopathology 2014; 104: 224-231.

68. Xu H, Wang R, Zhao Y, Fu ZQ, Qian G, Liu F. LesR is a novel upstream regulator that controls downstream Clp expression to modulate antibiotic HSAF biosynthesis and cell aggregation in Lysobacter enzymogenes OH11. Microb Cell Fact 2017; 16: 1-9.

69. Ramos JL, Martínez-Bueno M, Molina-Henares AJ, Terán W, Watanabe K, Zhang X, et al. The TetR family of transcriptional repressors. Microbiol Mol Biol Rev 2005; 69: 326-356.

70. Wang P, Chen H, Qian G, Liu F. LetR is a TetR family transcription factor from Lysobacter controlling antifungal antibiotic biosynthesis. Appl Microbiol Biotechnol 2017; 101: 3273- 
71. Koskiniemi S, Lamoureux JG, Nikolakakis KC, t'Kint de Roodenbeke C, Kaplan MD, Low DA, et al. Rhs proteins from diverse bacteria mediate intercellular competition. Proc Natl Acad Sci U S A 2013; 110: 7032-7037.

72. Raymann K, Bobay L-M, Doak TG, Lynch M, Gribaldo S. A genomic survey of Reb homologs suggests widespread occurrence of R-bodies in proteobacteria. G3 (Bethesda) 2013; 3: 505-

73. Matsuoka J, Ishizuna F, Kurumisawa K, Morohashi K, Ogawa T, Hidaka M, et al. Stringent Expression Control of Pathogenic R-body Production in Legume Symbiont Azorhizobium

74. Braun V. Energy-coupled transport and signal transduction through the gram-negative outer membrane via TonB-ExbB-ExbD-dependent receptor proteins. FEMS Microbiol Rev 1995; 16: $295-307$.

75. Tseng T-T, Tyler BM, Setubal JC. Protein secretion systems in bacterial-host associations, and

76. Green ER, Mecsas J. Bacterial Secretion Systems : An Overview. 2016; 1-19.

77. de Bruijn I, Cheng X, de Jager V, Expósito RG, Watrous J, Patel N, et al. Comparative

78. Ling J, Zhou L, Wu G, Zhao Y, Jiang T, Liu F. The AHL Quorum-Sensing System Negatively Regulates Growth and Autolysis in Lysobacter brunescens. Front Microbiol 2019; 10: 1-13.

79. McCarthy RR, Yu M, Eilers K, Wang Y-C, Lai E-M, Filloux A. Cyclic di-GMP inactivates T6SS and T4SS activity in Agrobacterium tumefaciens. Mol Microbiol 2019; 112: 632-648. 
589 80. Bleves S, Soscia C, Nogueira-Orlandi P, Lazdunski A, Filloux A. Quorum sensing negatively 590 controls type III secretion regulon expression in Pseudomonas aeruginosa PAO1. J Bacteriol 2005; 187: 3898 LP - 3902.

592 81. Ruwandeepika HAD, Karunasagar I, Bossier P, Defoirdt T. Expression and Quorum Sensing Regulation of Type III Secretion System Genes of Vibrio harveyi during Infection of Gnotobiotic Brine Shrimp. PLoS One 2015; 10: e0143935-e0143935.

82. Atkinson S, Goldstone RJ, Joshua GWP, Chang C-Y, Patrick HL, Cámara M, et al. Biofilm development on Caenorhabditis elegans by Yersinia is facilitated by quorum sensingdependent repression of type III secretion. PLoS Pathog 2011; 7: e1001250. 


\section{Figure legends}

601

602

603

604

605

606

607

608

609

610

Figure 1. Location of rpf genes in Lysobacter capsici AZ78. (a) Comparison of the clusters of genes involved in Diffusible Soluble Factors synthesis and perception in L. enzymogenes C3 and L. capsici AZ78. Gene names are given within the arrows. The corresponding accession number is given under each gene. (b) Bioassay showing DSF production ability (blue halo) of L. capsici AZ78 in comparison to Xanthomonas campestris pv. campestris 8004.

Figure 2. Effect of diffusible communication signals on the inhibitory activity of Lysobacer capsici AZ78 against (a) Pythium ultimum and (b) Rhodococcus fascians. Antioomycete and antibacterial activity is expressed as the mean value and standard error variation (percentage) of the reduction of the mycelium growth area of P. ultimum and R. fascians compared to the control (L. capsici AZ78 in not supplemented media), respectively. LeDSF3: 13-methyltetradecanoic acid $50 \mu \mathrm{M}$, IND: indole $500 \mu \mathrm{M}$, GLY: glyoxylic acid $0.01 \mu \mathrm{M}$, BUT: 2,3-butanedione $0.01 \mu \mathrm{M}, 3 \mathrm{HBA}$ : 3-hydroxybenzoic acid $30 \mu \mathrm{M}$, 4HBA: 4-hydroxybenzoic acid $50 \mu \mathrm{M}$, AHL: mix of $\mathrm{N}$-acyl homoserine lactones $20 \mu \mathrm{M}$. Each treatment included five replicates and data originating from two independent experiments were pooled. Different letters indicate significant letters according to Tukey's test $(\alpha=0.05)$.

Figure 3. Hierarchical clustering of Lysobacter capsici AZ78 genes differentially expressed (1,115 genes, Tables S5-S11 for gene expression data). LeDSF3: 13-methyltetradecanoic acid $50 \mu \mathrm{M}$, IND: indole $500 \mu \mathrm{M}$, GLY: glyoxylic acid $0.01 \mu \mathrm{M}$, BUT: 2,3-butanedione $0.01 \mu \mathrm{M}$, 3HBA: 3hydroxybenzoic acid $30 \mu \mathrm{M}, 4 \mathrm{HBA}$ : 4-hydroxybenzoic acid $50 \mu \mathrm{M}$, AHL: mix of N-acyl homoserine lactones $20 \mu \mathrm{M}$.

Figure 4. Radar plots of Lysobacter capsici AZ78 genes differentially expressed in response to diffusible communication signals. Annotated genes were classified in 20 functional categories: global metabolism (MET); carbohydrate metabolism (CM); energy metabolism (E); lipid metabolism (LM); nucleotide metabolism (NM); amino acid metabolism (AM); protein metabolism (PM); secondary 
metabolism (SM); DNA metabolism (DNA); RNA transcription and degradation (RNA/TR); translation $(\mathrm{T})$; growth $(\mathrm{G})$; oxidative stress $(\mathrm{OX})$; antagonism (AG); defence (D); transport, phosphotransferase systems and secretion (T); signal transduction and receptors (S); kinase/phosphatase (K); quorum sensing (QS); motility, chemotaxis, and biofilm (M). Only genes with $\mid \log 2$-fold change $\mid>1$ and p-value $<0.01$ were included. LeDSF3: 13-methyltetradecanoic acid $50 \mu \mathrm{M}$, IND: indole $500 \mu \mathrm{M}$, GLY: glyoxylic acid $0.01 \mu \mathrm{M}$, BUT: 2,3-butanedione $0.01 \mu \mathrm{M}, 3 \mathrm{HBA}$ : 3-hydroxybenzoic acid $30 \mu \mathrm{M}$, 4HBA: 4-hydroxybenzoic acid $50 \mu \mathrm{M}$, AHL: mix of N-acyl homoserine lactones $20 \mu \mathrm{M}$.

Figure 5. Heatmap of Lysobacter capsici AZ78 genes differentially expressed in response to diffusible communication signals and related to efflux pumps, type IV pilus, antagonism, cell-cell communication systems and secretion systems. HSAF: Heat Stable Antifungal Factor. VirB6 * represent duplicate gene pairs located through AZ78 genome. LeDSF3: 13-methyltetradecanoic acid $50 \mu \mathrm{M}$, IND: indole $500 \mu \mathrm{M}$, GLY: glyoxylic acid $0.01 \mu \mathrm{M}$, BUT: 2,3-butanedione $0.01 \mu \mathrm{M}, 3 \mathrm{HBA}$ : 3-hydroxybenzoic acid $30 \mu \mathrm{M}$, 4HBA: 4-hydroxybenzoic acid $50 \mu \mathrm{M}$, AHL: mix of N-acyl homoserine lactones $20 \mu \mathrm{M}$.

Figure 6. Schematic representation of Lysobacter capsici AZ78 response to diffusible communication signals. LeDSF3: 13-methyltetradecanoic acid; IND: indole; GLY: glyoxylic acid; BUT: 2,3-butanedione, 3HBA: 3-hydroxybenzoic acid; 4HBA: 4-hydroxybenzoic acid; AHL: Nacyl homoserine lactones. 
643

644

Table 1. Cell-cell communication genes present in the genome of Lysobacter capsici AZ78.

\begin{tabular}{cccccccc}
\hline $\begin{array}{c}\text { Gene } \\
\text { abbreviation }\end{array}$ & L. capsici AZ78 & \multicolumn{2}{c}{ L. enzymogenes C3 } & S. maltophilia K279a & \multicolumn{2}{c}{$\begin{array}{c}\text { X. campestris pv. } \\
\text { campestris ATCC 33913 }\end{array}$} \\
\hline rpfG & AZ78_0630 & GLE_2281 & $97 \%$ & Smlt2233 & $83 \%$ & XCC1854 & $80 \%$ \\
$r p f B$ & AZ78_0629 & GLE_2284 & $89 \%$ & Smlt2236 & $79 \%$ & XCC1858 & $74 \%$ \\
$r p f C$ & AZ78_3298 & GLE_2282 & $31 \%$ & Smlt2234 & $30 \%$ & XCC1856 & $29 \%$ \\
rpfF & AZ78_3297 & GLE_2283 & $35 \%$ & Smlt2235 & $34 \%$ & XCC1857 & $35 \%$ \\
trpC & AZ78_4108 & GLE_4270 & $87 \%$ & Smlt4309 & $66 \%$ & XCC0470 & $67 \%$ \\
qseB & AZ78_2946 & GLE_5247 & $93 \%$ & & & XCC3893 & $59 \%$ \\
qseC & AZ78_2945 & GLE_5248 & $82 \%$ & Smlt1421 & $29 \%$ & XCC3894 & $48 \%$ \\
xanB2 & AZ78_3466 & GLE_4979 & $84 \%$ & & & XCC4014 & $65 \%$ \\
lysR & AZ78_2901 & GLE_5229 & $90 \%$ & & & & \\
luxR & AZ78_4823 & GLE_1495 & $77 \%$ & Smlt1839 & $48 \%$ & & \\
\hline
\end{tabular}

645

646

647

648

649

650

651

652

653

654

\section{Tables}

Percent identity at amino acid level between putative cell-cell communication genes of $L$ capsici AZ78 and their orthologues in L. enzymogenes C3 (CP013140.1), Stenotrophomonas maltophilia K279a (AM743169.1), and Xanthomonas campestris pv. campestris ATCC $33913^{\mathrm{T}}$ (AE008922.1). Corresponding locus tag for each gene and organisms are given.

Table 2. Differentially expressed genes in Lysobacter capsici AZ78 in response to diffusible communication signals after $48 \mathrm{~h}$ incubation.

\begin{tabular}{llrrrrrrr}
\hline & LeDSF3 & IND & GLY & BUT & 3HBA & 4HBA & AHL \\
\hline \multirow{2}{*}{ UP- } & Genes with assigned function & 325 & 312 & 142 & 107 & 48 & 34 & 17 \\
REGULATED & Hypothetical proteins & 40 & 56 & 42 & 38 & 12 & 14 & 3 \\
& Total no. of genes & $\mathbf{3 6 5}$ & $\mathbf{3 6 8}$ & $\mathbf{1 8 4}$ & $\mathbf{1 4 5}$ & $\mathbf{6 0}$ & $\mathbf{4 8}$ & $\mathbf{2 0}$ \\
\hline \multirow{2}{*}{ DOWN- } & Genes with assigned function & 194 & 169 & 78 & 72 & 33 & 8 & 2 \\
\multirow{2}{*}{ REGULATED } & Hypothetical proteins & 77 & 66 & 30 & 20 & 8 & 2 & 2 \\
& Total no. of genes & $\mathbf{2 7 1}$ & $\mathbf{2 3 5}$ & $\mathbf{1 0 8}$ & $\mathbf{9 2}$ & $\mathbf{4 1}$ & $\mathbf{1 0}$ & $\mathbf{4}$ \\
\hline \multirow{3}{*}{ TOTAL } & Genes with assigned function & 519 & 481 & 220 & 179 & 81 & 42 & 19 \\
& Hypothetical proteins & 117 & 122 & 72 & 58 & 20 & 16 & 5 \\
& Total no. of genes & $\mathbf{6 3 6}$ & $\mathbf{6 0 3}$ & $\mathbf{2 9 2}$ & $\mathbf{2 3 7}$ & $\mathbf{1 0 1}$ & $\mathbf{5 8}$ & $\mathbf{2 4}$ \\
\hline
\end{tabular}

Each treatment was subjected to pairwise comparison with the untreated control. |Log2-fold change| $>1$ and p-value < 0.01 were chosen as cut-off values for differential gene expression. LeDSF3: 13-methyltetradecanoic acid $50 \mu \mathrm{M}$, IND: indole $500 \mu \mathrm{M}$, GLY: glyoxylic acid $0.01 \mu \mathrm{M}$, BUT: 2,3-butanedione $0.01 \mu \mathrm{M}, 3 \mathrm{HBA}$ : 3-hydroxybenzoic acid $30 \mu \mathrm{M}$, 4HBA: 4-hydroxybenzoic acid $50 \mu \mathrm{M}$, AHL: mix of N-acyl homoserine lactones $20 \mu \mathrm{M}$. 
a

\section{Lysobacter enzymogenes C3}

$2,498,081$

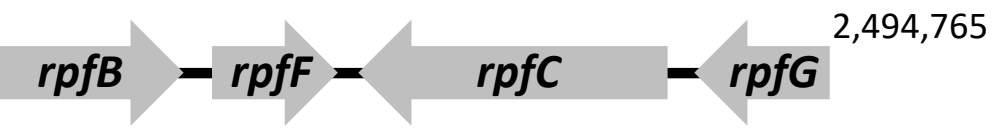

GLE_2284 GLE_2283 GLE_2282 GLE_2281

\section{Lysobacter capsici AZ78}

857,114

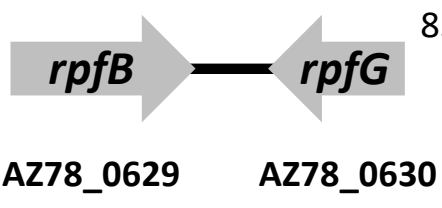

b

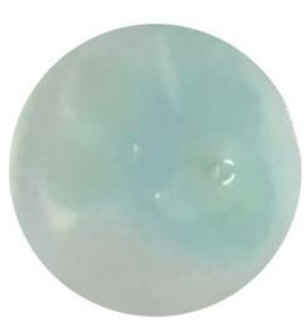

Lysobacter capsici AZ78
859,152
$3,947,548$
$r p f F=\quad r p f C$
$3,948,450$
AZ78_3298 AZ78_3297 

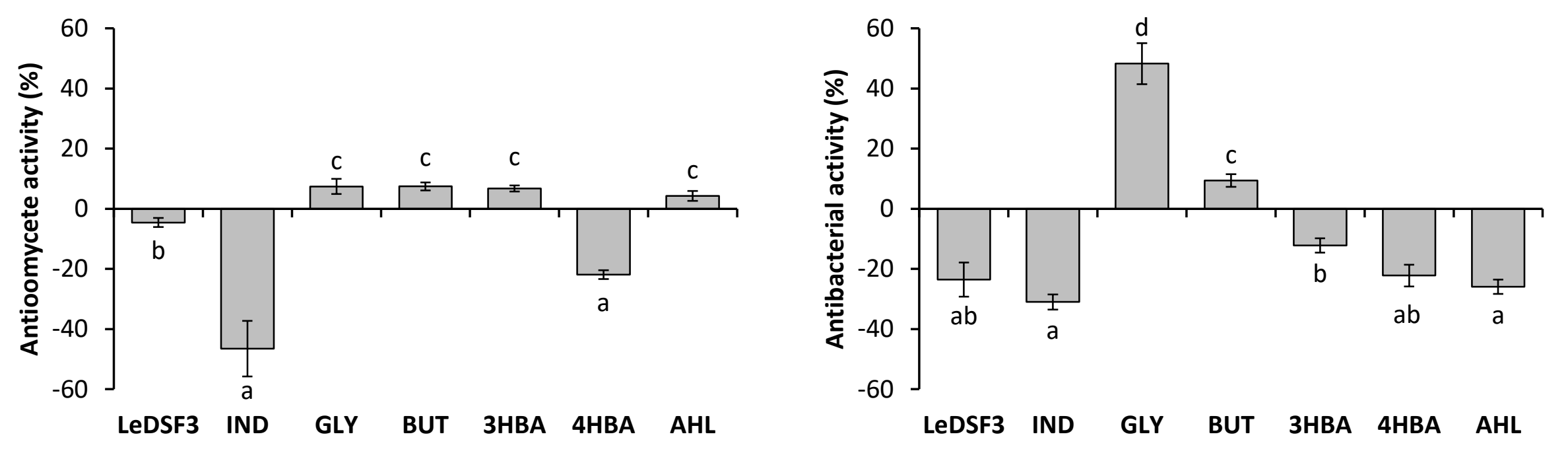


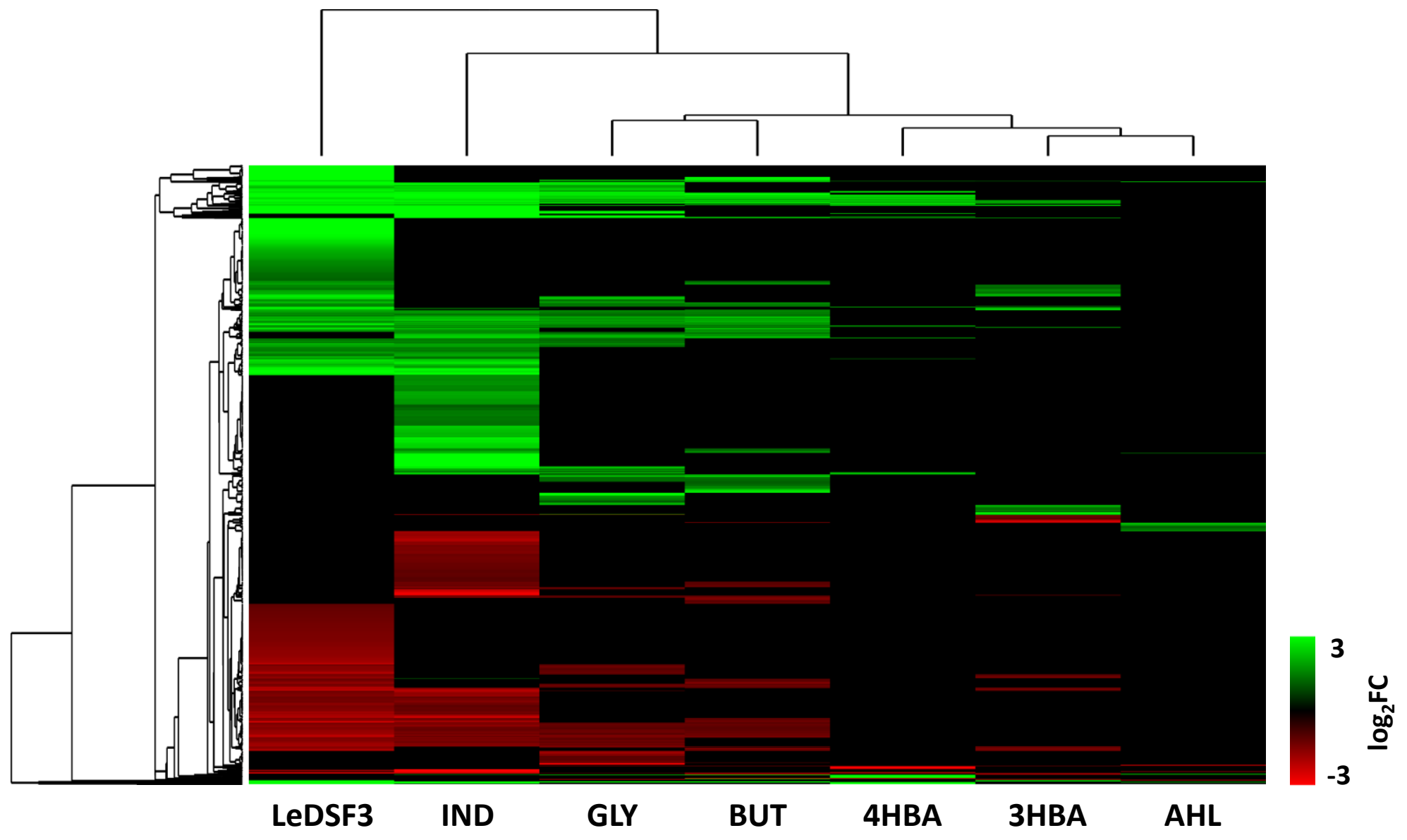




\section{UP REGULATED}

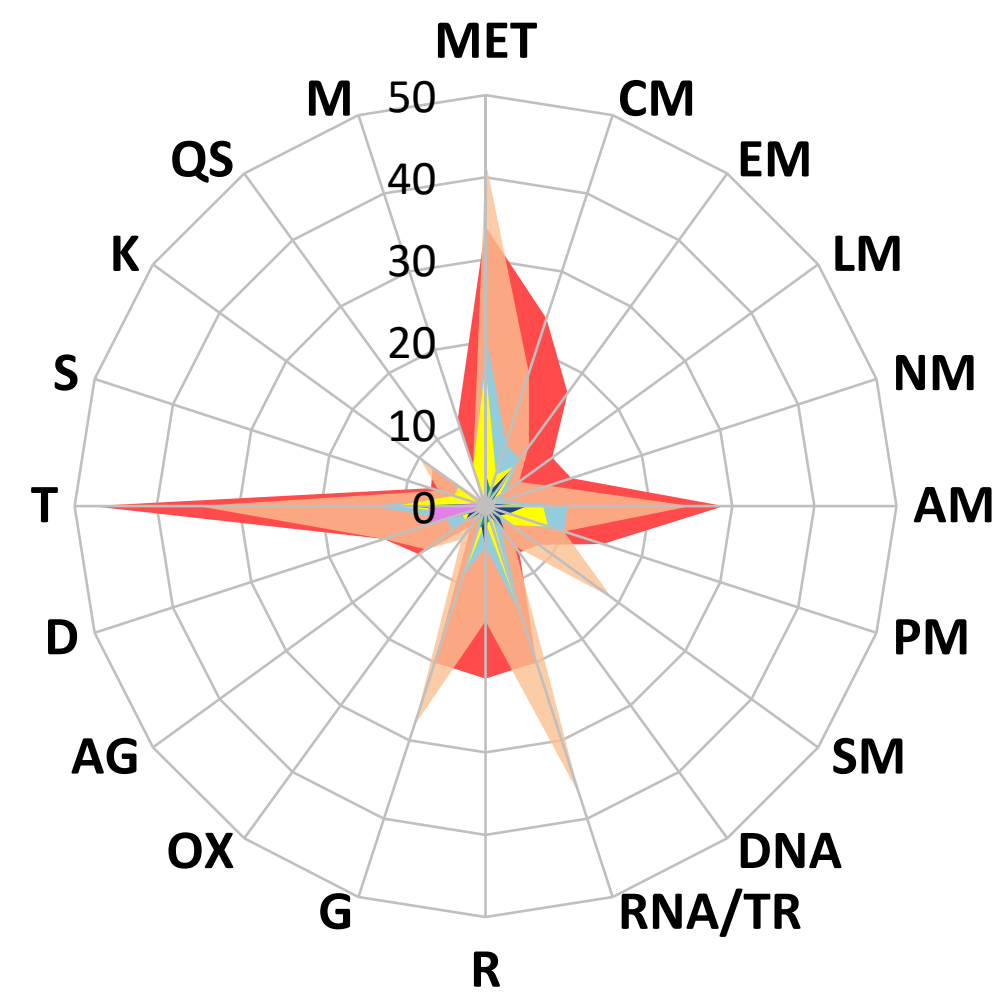

\section{DOWN REGULATED}

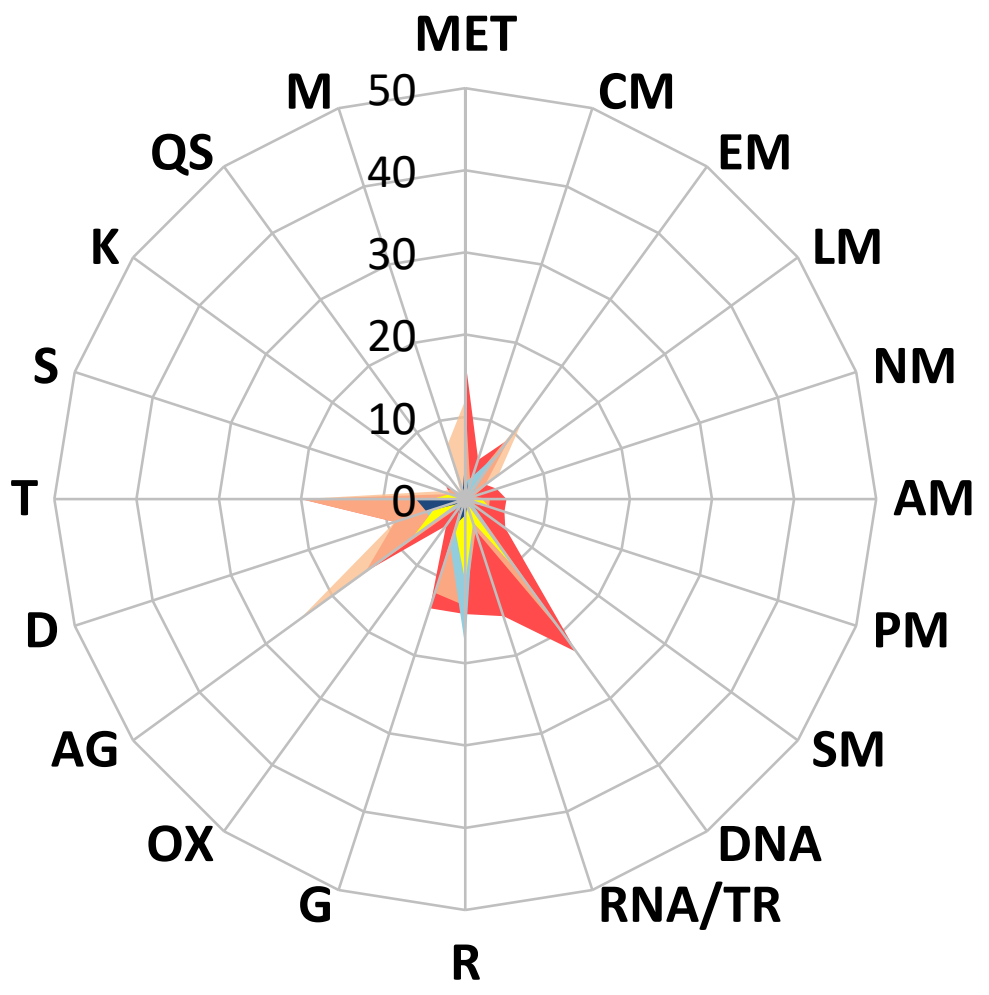




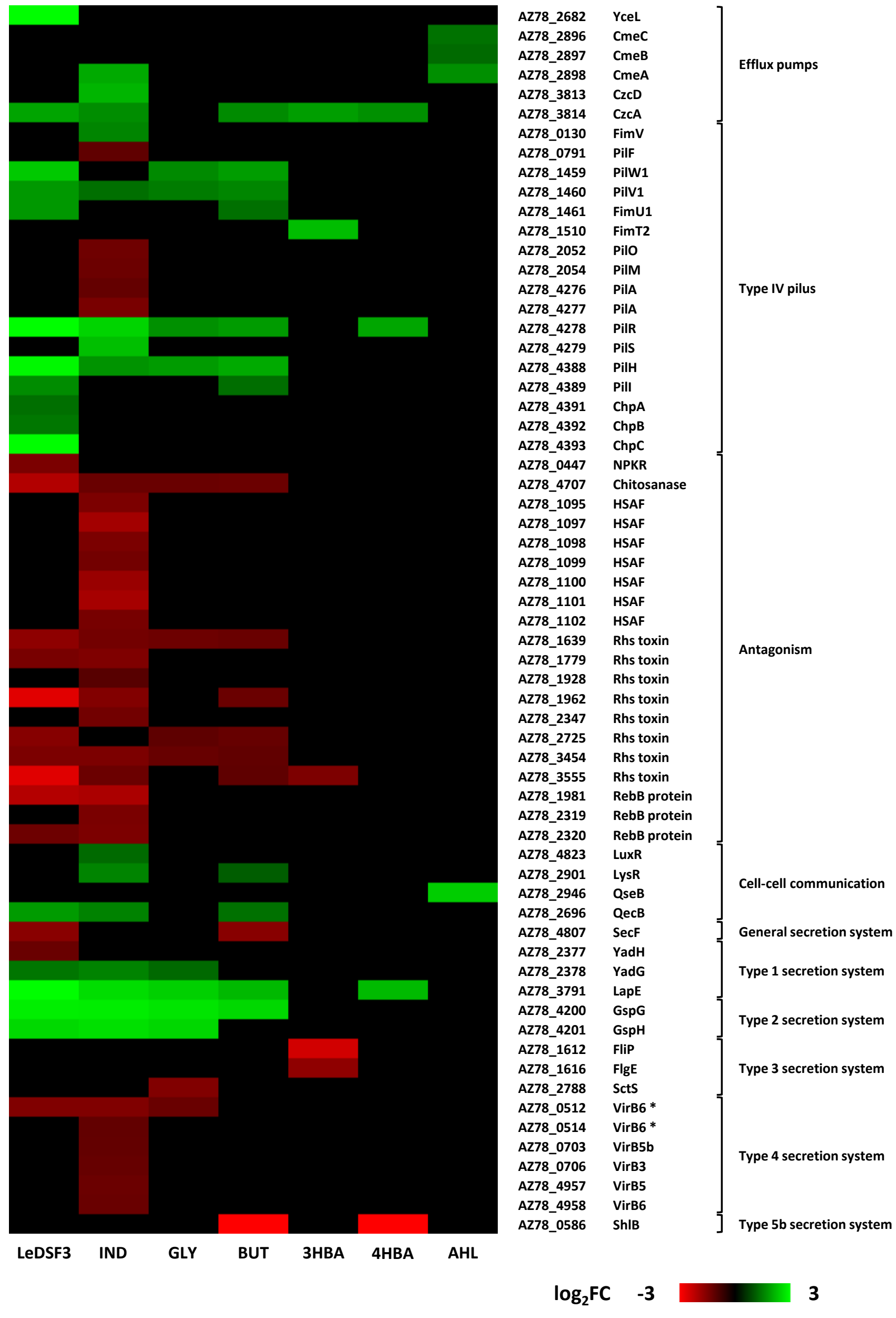




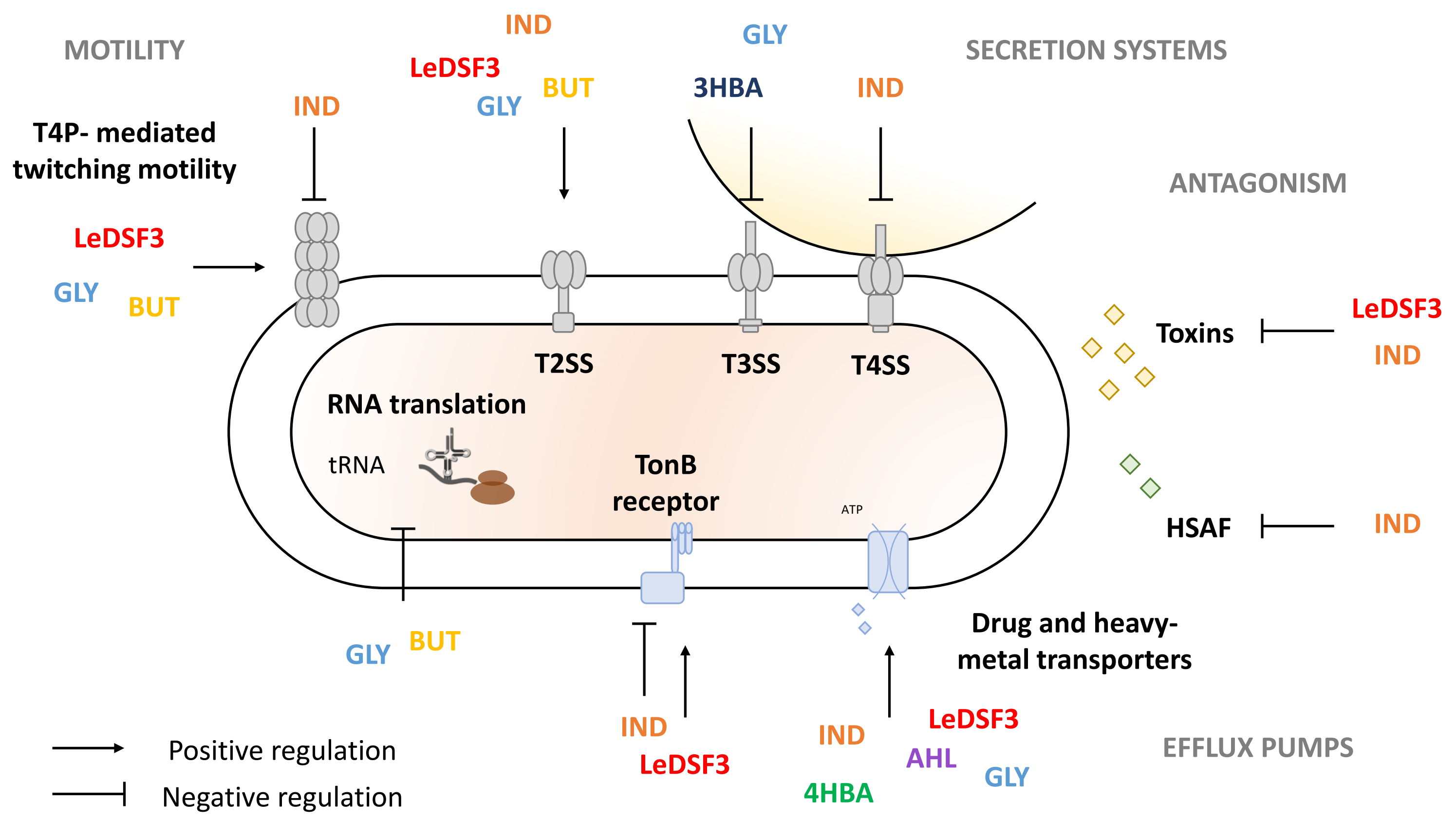

